・方法・

\title{
基于GIS的外来入侵植物调查规划与外业实施
}

\author{
陈 宏 ${ }^{1}$ 冼晓青 ${ }^{2}$ 邱荣洲 $^{3}$ 池美香 $^{3}$ 赵 健 $^{*}$ \\ 1 (福建省农业科学院数字农业研究所, 福州 350003) \\ 2 (中国农业科学院植物保护研究所, 北京 100193) \\ 3 (福建省农业科学院植物保护研究所, 福州 350012)
}

\begin{abstract}
摘要: 前期规划对外来入侵植物野外调查工作的顺利开展至关重要。本文将基于GIS的野外调查规划方法和入侵 植物调查技术规范相结合, 利用地图资源丰富、分析功能强大的LocaSpace Viewer和奥维互动地图, 形成有针对性 的野外调查规划方案。具体操作流程包括: (1)借助LocaSpace Viewer软件集成的高分辨率遥感数据和地理标注数据 选取调查区域; (2)根据调查规范, 通过该软件的空间分析和地理要素编辑功能, 规划调查地理网格、调查点、样地、 标准地和样方, 运用KML空间数据格式存储和分享调查规划信息; (3)在外业实施中使用移动版奥维互动地图软件 的数据管理和导航功能实现调查规划信息的导入、定位和导航。通过在福建、广西和云南三省开展技术方法的实 地应用与对比实验发现, 运用该方案的外业实施能够达到日平均调查 6 个样点, 采集调查数据 80 多条, 直线调查距 离 $180 \mathrm{~km}$, 调查点导航精度大于 $15 \mathrm{~m}$ 。对比实验表明基于规划调查比未规划调查在时间利用效率上提高 1 倍, 工作 效率得到显著提升。该方案为外来入侵植物的发生和危害等基础性调查工作提供了高效的技术支撑, 为生物多样 性野外调查规划提供了参考方案。
\end{abstract}

关键词: GIS; 外来入侵植物; 调查规划; 外业实施; LocaSpace Viewer

\section{Planning and conducting field surveys of invasive alien species based on GIS}

\author{
Hong Chen ${ }^{1}$, Xiaoqing Xian ${ }^{2}$, Rongzhou Qiu ${ }^{3}$, Meixiang Chi ${ }^{3}$, Jian Zhao ${ }^{1 *}$ \\ 1 Institute of Digital Agriculture Research, Fujian Academy of Agricultural Sciences, Fuzhou 350003 \\ 2 Institute of Plant Protection, Chinese Academy of Agricultural Sciences, Beijing 100193 \\ 3 Institute of Plant Protection, Fujian Academy of Agricultural Sciences, Fuzhou 350012
}

\begin{abstract}
Pre-planning plays a vital role in developing invasive alien plant surveys rapidly and conveniently in the field. This study combined survey technical standards of invasive alien plants and field survey planning methods based on GIS to develop a targeted field survey planning program. LocaSpace Viewer and Orvital maps, which provide rich map resources and powerful functions, were mainly applied. The detailed workflow was as follows: Firstly, the target areas for invasive alien plant field surveys were selected by integrating high-resolution remote sensing data and geographical annotation data in the LocaSpace Viewer; Secondly, according to standards of invasive alien plant field surveys, survey geographic grids, survey points, sample plots, standard-plots, and quadrats were established through the functions of spatial analysis and editing geographic elements in the LocaSpace Viewer, of which information were stored and shared in the KML format; Finally, positioning and navigation of survey points were implemented by Data Management and Navigation functions in the mobile terminal software of the Orvital map. Comparative experiments and applications of proposed technology and methodology have been carried out in Fujian, Guangxi, and Yunnan provinces. Results suggested that the developed technology and methods for planning invasive alien plant surveys and field operations based on GIS technology can help to fulfill surveys in 6 sample points and collect more than 80 records per day. The direct distance of daily invasive alien plant survey is approximately $180 \mathrm{~km}$. The navigation accuracy of survey points is better than $15 \mathrm{~m}$. Planning-based surveys have double the time utilization
\end{abstract}

收稿日期: 2017-11-26; 接受日期: 2018-01-18

基金项目：国家重点研发计划(2016YFC202105)和福建省科技重大专项(2017NZ0003-1)

* 通讯作者 Author for correspondence. E-mail: zhaojian@faas.cn 
rate of unplanned surveys. The technical route presented in this study greatly contributes to improving the efficiency of invasive alien plant surveys and provides powerful technical support for invasive alien plant survey in the field and insight into biodiversity field surveying.

Key words: GIS; invasive alien plants; survey planning; field operation; LocaSpace Viewer

随着全球化进程的推进，国际旅游、物流和贸 易往来日益频繁, 加快了物种迁徙和传播的速度, 造成外来入侵物种为害日益严重, 对生态环境构成 巨大威胁(万方浩等, 2002; 丁目军等, 2015)。野外调 查是外来入侵植物研究和管理的基础, 可为风险评 估、监测、预警、防控提供基础数据支撑。外来入 侵植物由于在个体、种群、生境、空间分布等方面 存在差异, 再加上调查工作还受到天气、环境、交 通、人员等因素的影响(McMaugh, 2013), 在开展多 种入侵植物调查和大量的数据采集工作之前进行 周密规划至关重要。

外来入侵植物分布具有典型的时空相关性, 地 理信息系统(geographic information system, GIS)因 在时空数据管理、分析和展示方面的优势, 为调查、 研究其空间分布和扩散规律提供了新思路和新方 法(孙文涛和刘雅婷, 2010)。国内已有学者对野外调 查中涉及的地理信息管理、路线设计和导航方面提 出技术方案, 将物种野外调查涉及的信息抽象为 点、线和面等地理信息进行管理和路线设计(陈涁, 2016)。但在实际工作中, 调查目的和调查对象的差 异往往要求不同的调查方法。在不同的调查技术规 程中, 点、线和面等地理信息被赋予不同的含义, 这将直接关系到调查数据的管理、分析与应用。因 此, 调查规划必须紧密结合具体的应用场景, 根据 调查规程明确点、线、面等地理信息的科学含义。 此外, 不同的调查工作对调查点及周边范围、样方 大小、调查点间隔等都提出具体的量化指标(方精云 等, 2009)。根据这些指标运用GIS空间分析方法进 行调查点选址, 能够精准划定调查范围的边界, 优 化调查点布局, 避免依靠人为经验造成调查点在空 间分布上的随机性，降低调查数据的质量。

本文以外来入侵植物调查为应用场景, 根据调 查技术与方法规范(万方浩等, 2011), 提出基于 GIS 的外来入侵植物调查规划和外业实施技术方案(附 录1)。通过GIS软件提供的遥感本底和地理标注数 据识别调查区域生态系统类型, 运用空间分析和地 理要素编辑功能划分调查地理网格, 规划调查点、
样地、标准地和样方, 利用KML (Keyhole标记语言, Keyhole markup language)数据格式共享调查规划信 息。在外业调查实施中利用移动导航软件对调查规 划信息进行解析、定位和导航，实现外来入侵物种 野外调查的精准实施。在福建、广西、云南三省开 展技术应用, 并在广西调查中进行对比实验, 验证 方法的科学性、准确性和高效性。

\section{1 技术路线与软件工具}

\section{1 技术路线}

外来入侵植物调查分 3 个阶段：前期准备、调查 规划和外业实施。前期准备需要收集相关的信息和 资料; 调查规划是根据调查规程和要求规划调查 点、样地、标准地、样方和调查路径; 外业实施是 利用规划信息进行调查路径导航，开展调查工作。

\section{2 使用软件工具和数据交互格式}

\subsubsection{LocaSpace Viewer}

LocaSpace Viewer是由苏州中科图新网络科技 有限公司开发的一款专业的免费三维数字地球软件 (http://www.3dyuanjing.com/Viewer/LocaSpaceViewe r.zip)。与传统的 Google Earth 相比, LocaSpace Viewer功能上的优势在于集成了丰富的在线地图资 源, 包括街道图、遥感图和专题图等, 能够更加全 面、详细地了解调查区域的生态环境; 提供了缓冲 区分析等多种空间分析工具和复杂地理要素编辑 功能, 无需借助其他软件就能完成调查规划数据的 分析与处理, 降低了对软件操作的要求, 避免了不 同软件间数据转换和传输造成的误差; 支持多种数 据格式导入、汶览和转换, 为后续调查数据汇总、 分析提供了便利。LocaSpace Viewer与Google Earth 在数据资源与功能方面的对比如表 1 所示。

\subsection{2 奥维互动地图}

奥维互动地图是由北京元生华网软件有限公 司开发的集成多种在线地图资源的跨平台地图汶 览器 (http://www.ovital.com/download/)。与流行的 OruxMaps地图汶览软件相比, 奥维互动地图的优 势在于支持多种移动终端操作系统; 中文界面布局 
简洁、操作简单; 国内地图资源丰富且在线刷新速 度快; 拥有强大的地理信息展现技术, 支持多种数 据格式的导入导出, 方便规划数据的汶览; 支持中 国区域的线路、地理位置、行政区域、经纬度等多 样化信息的检索和路径导航，便于野外调查导航。 奥维互动地图与OruxMaps在数据资源和功能方面 的对比如表2所示。

\subsubsection{KML数据格式}

KML是一种基于XML (可扩展标记语言, extensible markup language)语法和文件格式的数据文 件, 可用来描述和保存地理数据, 包括点、线、面、 多边形、多面体和模型等, 可应用于Google Earth相 关软件及第三方支持 $\mathrm{KML}$ 数据格式的软件, 用于 地理数据的展示与分析。KML是地理标识语言 (geography markup language)的一个范例, 并已被接 纳为开放地理信息系统编码标准(open geospatial consortium KML encoding standard) (马立广和曹彦 荣, 2010; 杨立法, 2013)。KML数据格式适合于外来 入侵植物调查规划数据的存储与共享, 可作为规划 信息分发与协同的交互载体。

\section{2 外来入侵植物调查规划操作}

外来入侵植物调查规划的总体思路是把调查 区域划分为 $35 \mathrm{~km} \times 40 \mathrm{~km}$ 的地理网格作为调查的 基本空间单元，在每个地理网格内沿特定的路线, 按照最有可能传入和暴发的入侵物种名单, 针对各 个重要生态系统类型，如公园、自然保护区、公共 绿地、花卉市场、湖泊和溪流、农田、果园、林场、 菜地、保护地，以及港口和重要货物集散中心及周 边地区，根据调查规范对调查点、样地、标准地和 样方等调查规划要素的设置指标, 利用GIS空间分 析结果, 合理篮选并确定调查规划要素的位置。调 查规划要素之间的空间关系如图1所示。

\section{1 前期准备}

系统收集与调查任务相关的基础数据和资料, 包括：与调查区域相关的植被、土壤、气候等自然 环境资料，明确当地主要生态环境类型；调查区域 农林资源情况; 与调查区域相关的外来入侵植物已 有的调查资料和历史记录，建立调查区域外来入侵 植物名录与生物学描述; 与调查区域相关的社会经

表1 LocaSpace Viewer与Google Earth数据资源与功能对比

Table 1 Comparison of LocaSpace Viewer with Google Earth in data resources and functions

\begin{tabular}{|c|c|}
\hline & LocaSpace Viewer \\
\hline $\begin{array}{l}\text { 在线地图资源 } \\
\text { Online map resources }\end{array}$ & $\begin{array}{l}\text { Google卫星影像、Google历史卫星影像、Google地形、天地图中 卫星影像、历史卫星影像、地形。Google satellite } \\
\text { 国地图、天地图分省地图、天地图卫星影像、Bing地图、Bing卫 images, Google historical satellite images, Google } \\
\text { 星影像、气象图、中国地质图、中国植被覆盖图、中国水系图。 terrain data. } \\
\text { Google satellite images, Google historical satellite images, Google } \\
\text { terrain data, MAPWORLD map of China, MAPWORLD provincial } \\
\text { map, MAPWORLD satellite images, Bing map, Bing satellite images, } \\
\text { Meteorological map, Geological map of China, Vegetation coverage } \\
\text { map of China, River map of China. }\end{array}$ \\
\hline $\begin{array}{l}\text { 地图标注 } \\
\text { Map annotation data }\end{array}$ & $\begin{array}{l}\text { 集成天地图标注, 覆盖全国的各级行政中心、居民点、兴趣点、各 提供省份及人口聚集地、省界、主要道路的标注, 但 } \\
\text { 级公路、水系、铁路等要素。Integrating MAPWORLD annotation 不完整。针对指定区域的标注需要导入KML地标文 } \\
\text { data, and important elements covering all levels of the country's ad- 件。Providing incomplete annotation data of different } \\
\text { ministrative centers, settlements, points of interest, all levels of roads, provinces, population centers, provincial boundaries } \\
\begin{array}{ll}\text { waterways and railways. } & \text { and the main roads. Placemark files in KML (Keyhole } \\
& \text { markup language) format for specific area is required } \\
& \text { to be imported into Google Earth. }\end{array}\end{array}$ \\
\hline $\begin{array}{l}\text { 支持数据类型 } \\
\text { Supported data types }\end{array}$ & $\begin{array}{l}\text { KML数据、影像数据、地形数据、CAD数据、矢量数据、GPS数 KML数据、影像数据、GPS数据。KML data, Image } \\
\text { 据。KML data, Image data, Terrain data, AutoCAD drawing database, data, GPS data. } \\
\text { Vector data, GPS data. }\end{array}$ \\
\hline $\begin{array}{l}\text { 分析功能 } \\
\text { Analysis functions }\end{array}$ & $\begin{array}{l}\text { 缓冲区分析、通视分析、可视域分析、填挖分析、剖面分析、距 距离测量、面积测量。Distance measurement, Area } \\
\text { 离测量、面积测量、三角测量。Buffer analysis, Visibility analysis, measurement. } \\
\text { Visible range analysis, Fill analysis, Profile analysis, Distance meas- } \\
\text { urement, Area measurement, Triangulation. }\end{array}$ \\
\hline $\begin{array}{l}\text { 编辑功能 } \\
\text { Editing functions }\end{array}$ & $\begin{array}{l}\text { 添加地标、绘制线、绘制面、绘制矩形、要素编辑工具。Add 添加地标、添加多边形、添加路径。Add placemark, } \\
\text { placemark, Draw line, Draw surface, Draw rectangle, Feature editing Add polygon, Add path. } \\
\text { tools. }\end{array}$ \\
\hline $\begin{array}{l}\text { 特色功能 } \\
\text { Special features }\end{array}$ & $\begin{array}{l}\text { 影像下载、地形下载、提取高程、数据转换、三维特效。Download } \\
\text { satellite image and terrain data, Extract elevation, Data conversion, } \\
\text { Three-dimensional effects. }\end{array}$ \\
\hline
\end{tabular}


表2 奥维互动地图与OruxMaps数据资源与功能对比

Table 2 Comparison of Ovital map with OruxMaps in data resources and functions

\begin{tabular}{ll}
\hline & 奥维互动地图 Ovital map \\
\hline 在线地图资源 & OruxMaps \\
Online map resources & Google地罗、国内外地图资源, 包括: 百度地图、搜狗地图、集成在线地图多为国外地图, 需要通过修改地图配 \\
& 形图、Bing卫星图、OpenCycle地形图。Integrate more map foreign online maps; the users need to modify the map \\
& resources at home and abroad, including Baidu Map, Sogou configuration file to increase the domestic online maps. \\
& Map, Google Map, Google satellite images, Google satellite \\
& images with labels, Google terrain data, Bing satellite images,
\end{tabular}

支持操作系统

Android, IOS (iPhone/ iPad), Windows, WindowsPhone

Android

Supported operating system

支持数据类型

KML, KMZ, GPX, PLT, DXF, DWG, TXT, CSV, SHP

Supported data types

软件界面

Software surface

操作体验

Operating experience
中文操作界面简洁 Simple Chinese interface

操作简单, 完全满足野外调查的数据浏览和导航需要。提供丰富的户外地图浏览功能, 但需要一定的学习 Simple operation; fully meet the needs of data browsing and 才能熟练操作。Have rich functionalities of outdoor navigation in field survey.

\section{KML, SHP, GPX}

界面功能按钮丰富，部分英文菜单。Rich interface function with buttons partly in English. map browsing, but the users are required to learn.

特色功能 Special features 数据网络存储 Provide network data storage

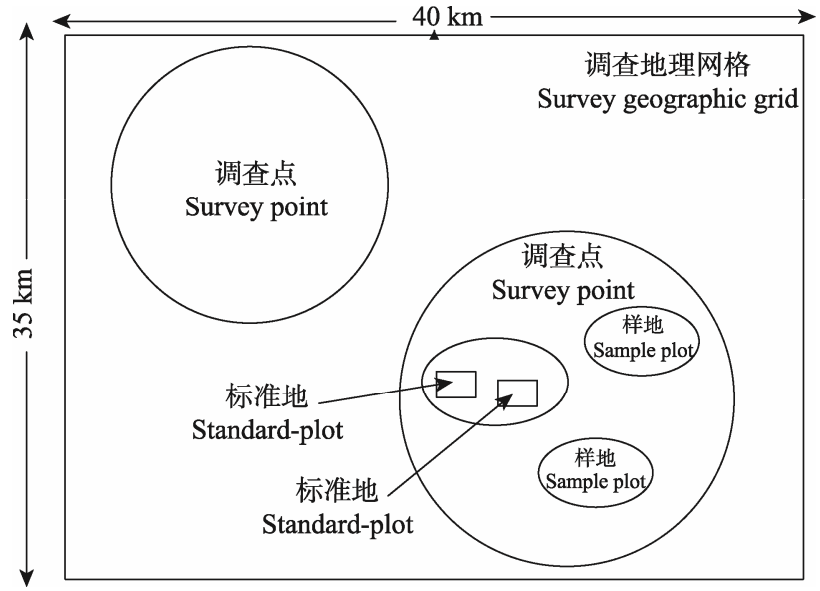

图1 外来入侵植物调查规划要素空间关系

Fig. 1 Spatial relationship of different planning elements for invasive alien plants survey

济状况等。此外还应明确调查任务、时间安排与人 员分工情况，便于统筹规划调查工作。

\section{2 调查区域地理网格划分}

应用LocaSpace Viewer软件将调查区域划分为 一定数量的地理网格 $(35 \mathrm{~km} \times 40 \mathrm{~km})$, 以此作为基 本单位进行调查数据记录。

软件操作步骤: (1)通过“操作 $\rightarrow$ 绘制矩形”功能, 标记调查区域; (2)在弹出的矩形属性窗口中填写地 理网格名称, 在“样式”分页栏中点击“纹理贴图”弹 出纹理参数设置窗口; (3)在纹理参数设置窗口中, 横向尺寸类型和纵向尺寸类型都设置成“纹理长度 (m)”, 根据入侵植物调查地理网格设置的要求, 横
向值设置成 $40,000 \mathrm{~m}$, 纵向值设置成 $35,000 \mathrm{~m}$, 横 向起始偏移量和纵向起始偏移量都设置成 0.5 , 纹 理路径选择附录 2 提供的十字透明背景纹理, 点击 “确定”按钮完成调查地理网格设置, 调查地理网格 设置如图2(A)所示。

\section{3 排查规划}

外来入侵植物排查是根据排查对象名单, 在调 查区域内开展调查, 以确定名单中入侵植物的发 生、分布情况。每个地理网格同一类型生态系统设 置 5 个调查点, 每个调查点设置2-3个样地。

以调查地理网格内高速公路收费站作为调查 点, 拟在其周边 $1 \mathrm{~km}$ 范围内的农田生态系统设置 2-3个样地开展排查, 在LocaSpace Viewer软件中进 行调查规划的操作步骤如下: (1)借助遥感影像和地 图标注在调查区地理网格内寻找高速公路收费站, 或者通过软件“搜索”功能搜索“高速收费站”，确定 调查点位置; (2)通过“操作 $\rightarrow$ 添加地标”功能, 标记 调查点; (3)通过“分析 $\rightarrow$ 创建缓冲区”功能，或者在 “场景数据” 菜单中右键点击调查点, 通过弹出菜单 中的“创建缓冲区”功能，创建宽度为 $1 \mathrm{~km}$ 的缓冲 区; (4)通过解析遥感影像在缓冲区内䇻选合适的 农田调查样地; (5)通过“操作 $\rightarrow$ 添加地标”功能, 标 记样地; (6)重复执行““(4) $\rightarrow(5)$ ””操作, 直到样地数 量满足排查需要; (7)将规划信息保存为KML文件。 设置的调查样地应考虑道路通达性且方便调查路 径规划。 


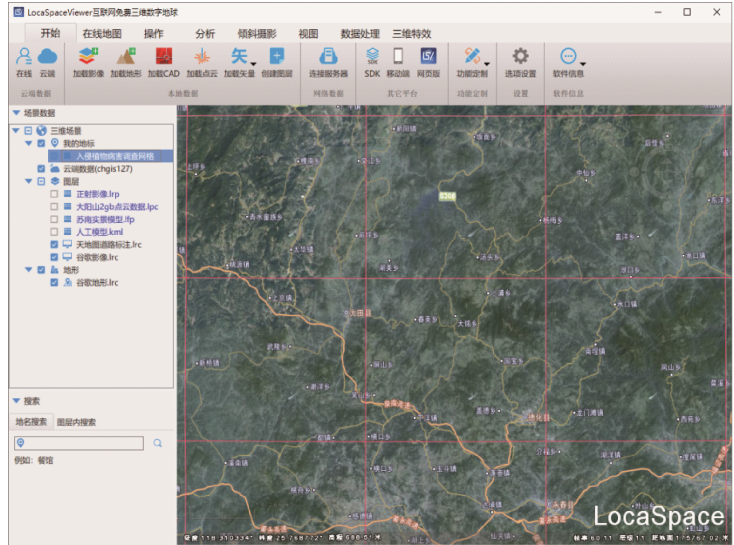

(A) 调查地理网格 Geographic grids for survey

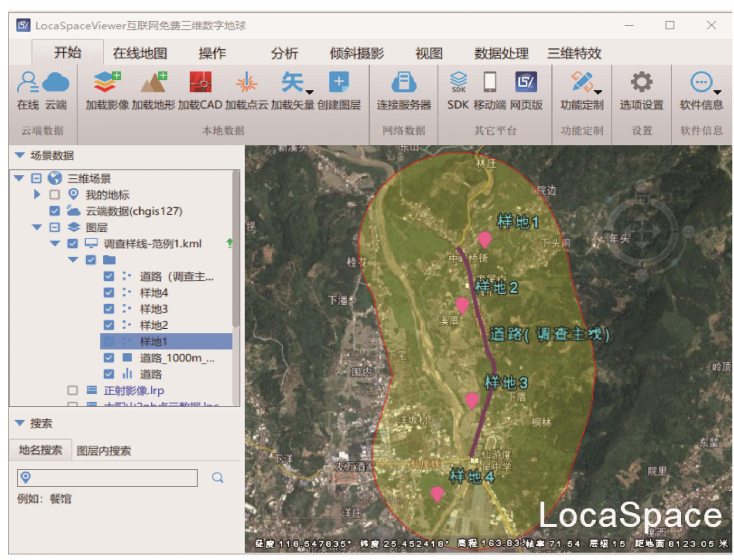

(C) 普查规划范例 Example of overall survey planning

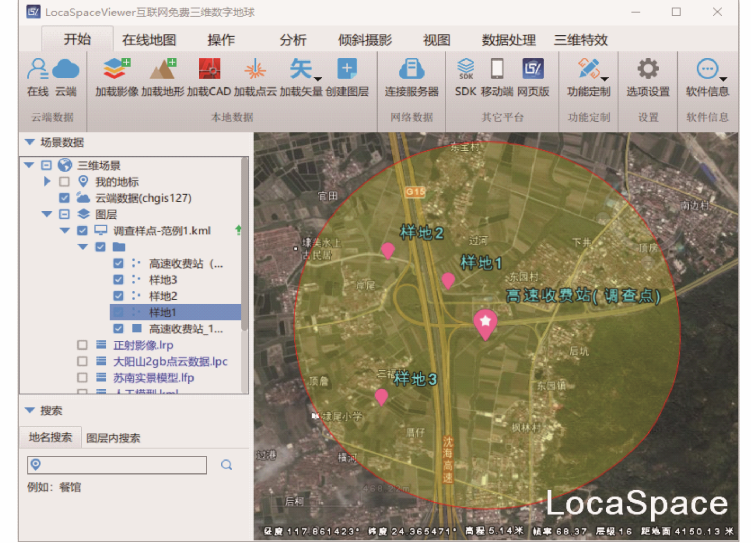

(B) 排查规划范例 Example of screening planning

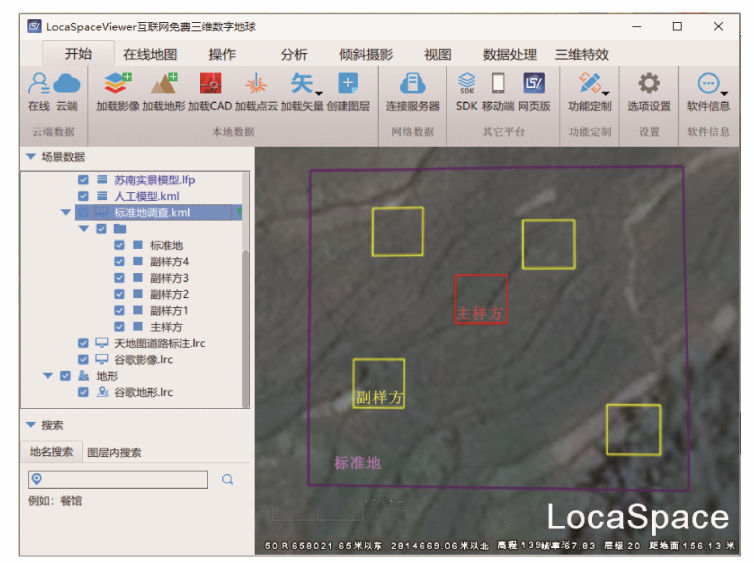

(D) 标准地调查规划范例 Example of standard-plot survey planning

\section{图2 外来入侵植物调查规划软件操作示意}

Fig. 2 Sample screen shots of invasive alien plants with survey planning software

此外, 还可以通过“分析 $\rightarrow$ 距离测量”功能, 测 算各调查点之间的距离, 保证调查点的间隔和空间 密度符合调查要求, 排查规划范例如图2(B)所示。

\section{4 普查规划}

外来入侵植物普查是在每一个地理网格内按 不同方向选择几条具有代表性的线路, 在每条线路 中针对生态系统的类型, 采取大范围、多点调查的 方式进行调查。每种生态系统类型选取多个具代表 性的样地进行调查。每1-5 km设置一个样地, 也可 根据具体情况调整调查样地分布的密度。

以调查地理网格内主要道路为代表性线路，拟 在其沿线周边 $1 \mathrm{~km}$ 范围内平均间隔1-5 km的农田 生态系统设置样地, 在LocaSpace Viewer软件中进 行调查规划的操作步骤如下: (1)借助遥感影像和地 图标注在调查地理网格内寻找适合的调查道路; (2) 通过 “操作 $\rightarrow$ 绘制线”功能, 标记所选道路作为调查 主线; (3)通过“分析 $\rightarrow$ 创建缓冲区”功能，或者在“场
景数据” 菜单中右键点击调查主线, 通过弹出菜单 中的“创建缓冲区”功能，创建宽度为 $1 \mathrm{~km}$ 的缓冲区; (4)通过解析遥感影像在缓冲区内的农田篮选合适 的调查样地; (5)通过“操作 $\rightarrow$ 添加地标”功能标记样 地; (6)重复执行““(4) $\rightarrow(5)$ ”操作, 直到样地数量满足 调查需要; (7)将规划信息保存为KML文件。普查规 划范例如图2(C)所示。

\section{5 标准地调查规划}

标准地调查是在普查的基础上, 针对重要生态 系统选择具有代表性的样地设置标准地, 在标准地 内开展进一步的样方调查。标准地选择必须或多或 少受到过人为或其他因素干扰, 样地内至少有 1 种 外来入侵植物, 标准地所在的植物群落尽可能包括 周围范围内其他植物的多样性和丰度, 还有可能的 各种环境或人为干扰。每个地理网格内如果生境相 对单一、群落类型一致，一般设置 1 个标准地即可; 如生境不同可按类型设置几个标准地; 如果某个地 
理网格内的生态系统很少或几乎没有受到人为的 干扰，该网格内可以不设标准地。

在每个标准地根据外来入侵植物的分布设置 1 个主样方, 以代表不同生态梯度。主样方设定后, 分别在其 4 个对角线上设置形状大小与主样方相同 的副样方, 用以计算目标入侵植物的出现频度, 修 正典型取样过程中可能造成的误差。主样方以及 4 个副样方的大小根据样方优势植物生活型进行设置: 乔木 $20 \mathrm{~m} \times 20 \mathrm{~m}$, 灌木 $10 \mathrm{~m} \times 10 \mathrm{~m}$, 草本 $2 \mathrm{~m} \times 2$ $\mathrm{m}$ 。在特殊情况下, 可以将 1 个主样方和 4 个副样方 设置在一条直线上, 适当延长主、副样方的距离。

以在农田生态系统的标准地内设置灌木样方 为例, 在LocaSpace Viewer软件中操作步骤如下: (1) 根据普查结果在拟开展调查的区域内, 通过“操作

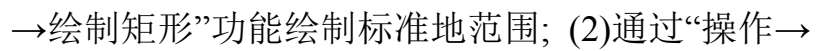
绘制矩形”功能, 绘制 $10 \mathrm{~m} \times 10 \mathrm{~m}$ 主样方矩形要素, 在矩形的“属性样式”窗口中选择“整体平移”功能, 将主样方矩形要素平移到标准地中央拟开展调查 的位置; (3)通过“操作 $\rightarrow$ 绘制矩形”功能, 绘制 $10 \mathrm{~m} \times$ $10 \mathrm{~m}$ 副样方矩形要素, 在矩形的“属性样式”窗口中 选择“整体平移”功能, 将副样方矩形要素平移到主 样方对角线拟开展调查的位置; (4)重复执行步骤 (3)操作, 完成余下的 3 个副样方设置; (5)将所有规 划信息另存为KML文件。标准地调查规划范例如图 2(D)所示。

\section{3 外业实施}

在实施大规模外业前, 可预先制定一套编码规 则, 对调查点、样地、标准地等规划要素进行唯一 性编号。为了方便任务分配与工作协同, 不同的调 查小组可根据分工使用不同样式标记，保存成不同 的KML文件。KML文件可以通过数据线、网络传 输、蓝牙对传等方式从电脑端发送到每个调查移动 终端。

在野外调查中使用的移动终端必须具备GPS 导 航功能, 并安装好奥维互动地图软件。调查导航前 先通过软件“收藏 $\rightarrow$ 菜单 $\rightarrow$ 导入导出 $\rightarrow$ 从文件导入” 功能在地图界面中汶览调查规划数据。在奥维互动 地图浏览器中进行调查导航的步骤如下: (1)在地图 中选择前往的调查样地，在弹出菜单中单击“导航” 按钮, 选择交通方式——软件提供了驾车、公共交 通和步行3种模式; (2)点击“搜索”按钮, 计算出从当 前位置前往调查样地的路线; (3)点击“导航”按钮开 始导航。在标准地调查中, 可以利用软件导航到调 查样方的四角点, 现场设置标记明确范围, 便于后 续开展重复调查。外业实施软件使用部分功能截图 如图3所示。

\section{4 应用与对比实验}

\section{1 调查规划应用}

依托国家重点研发计划“主要入侵生物的动态

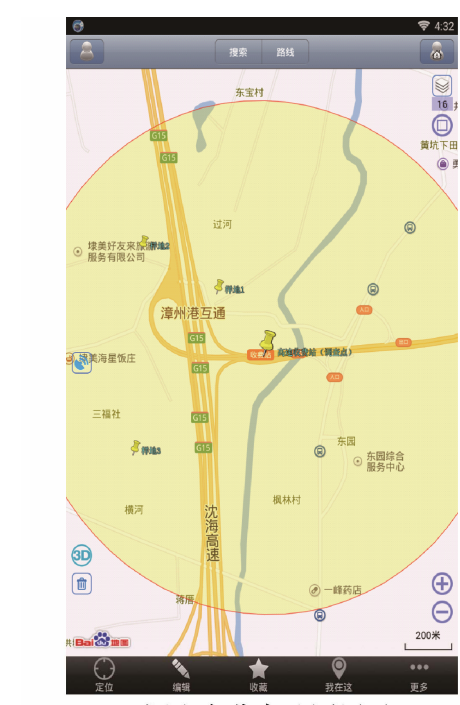

调查点分布地图展示 Survey points distribution shown on the map

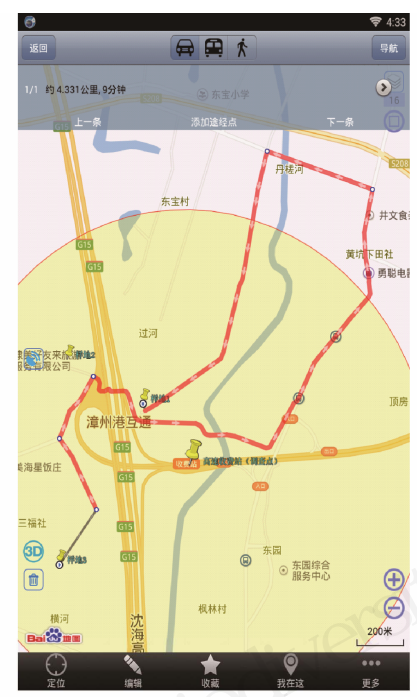

调查路径规划

Survey path planning

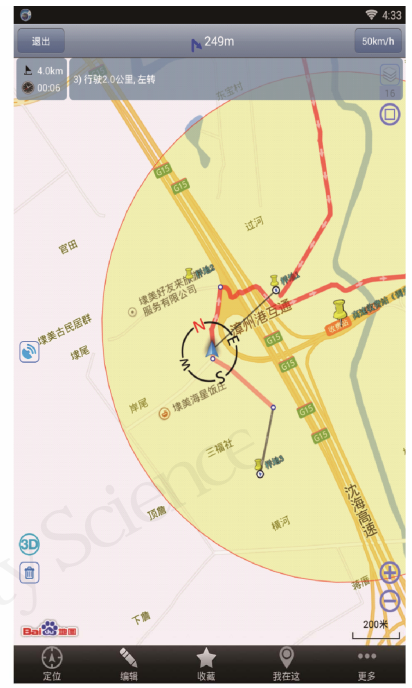

调查路径导航 Survey path navigation

图3 外业实施中奥维互动地图使用功能截图

Fig. 3 Function screen shots of Ovital map used in field operation 
表3 2017年3-5月福建、广西、云南三省入侵植物普查工作统计

Table 3 Statistics of invasive alien plants survey in Fujian, Guangxi and Yunnan provinces between March and May, 2017

\begin{tabular}{|c|c|c|c|c|c|c|c|c|c|}
\hline \multirow[t]{2}{*}{$\begin{array}{l}\text { 省份 } \\
\text { Province }\end{array}$} & \multirow[t]{2}{*}{$\begin{array}{l}\text { 调查日期 } \\
\text { Survey date } \\
\text { (Month/day) }\end{array}$} & \multicolumn{3}{|c|}{$\begin{array}{l}\text { 规划普查样地数量 } \\
\text { Number of planned } \\
\text { survey sample plots }\end{array}$} & \multicolumn{3}{|c|}{ 调查范围 Survey area } & \multicolumn{2}{|c|}{$\begin{array}{l}\text { 外来入侵植物普查数据统计 } \\
\text { Statistics of invasive alien } \\
\text { plants survey }\end{array}$} \\
\hline & & $\begin{array}{l}\text { 农田 } \\
\text { Farmland }\end{array}$ & $\begin{array}{l}\text { 湿地 } \\
\text { Wetland }\end{array}$ & $\begin{array}{l}\text { 港口 } \\
\text { Port }\end{array}$ & $\begin{array}{l}\text { 覆盖县域数量 } \\
\text { Number of cov- } \\
\text { ered counties }\end{array}$ & $\begin{array}{l}\text { 覆盖地理网格数量 } \\
\text { Number of geographic } \\
\text { grids }\end{array}$ & $\begin{array}{l}\text { 调查直线距离 } \\
\text { Direct distance } \\
\text { of survey }(\mathrm{km})\end{array}$ & $\begin{array}{l}\text { 调查数据量 } \\
\text { Number of } \\
\text { records }\end{array}$ & $\begin{array}{l}\text { 调查涉及种类 } \\
\text { Number of } \\
\text { species }\end{array}$ \\
\hline 福建 Fujian & $4 / 23-25,5 / 6-8$ & 40 & 0 & 0 & 27 & 29 & 1,100 & 590 & 40 \\
\hline 广西 Guangxi & $3 / 26-27$ & 10 & 0 & 3 & 7 & 7 & 330 & 82 & 28 \\
\hline 云南 Yunnan & $4 / 12-13$ & 6 & 2 & 0 & 7 & 7 & 426 & 132 & 28 \\
\hline
\end{tabular}

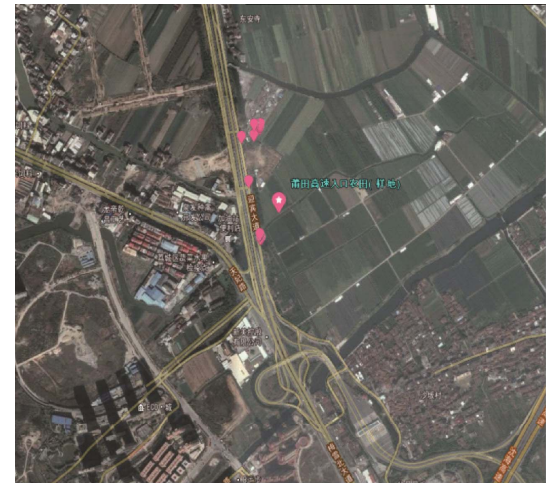

农田 Farmland

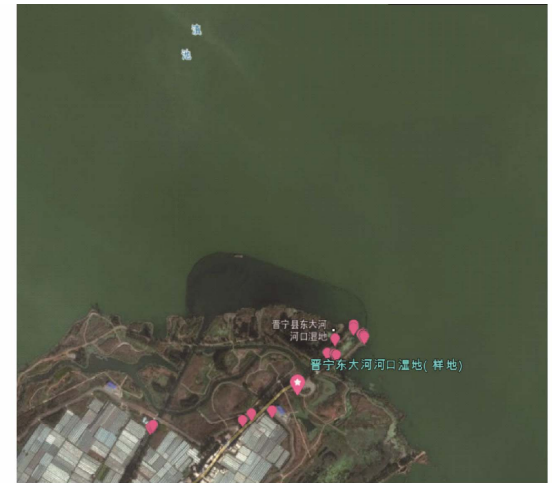

湿地 Wetland

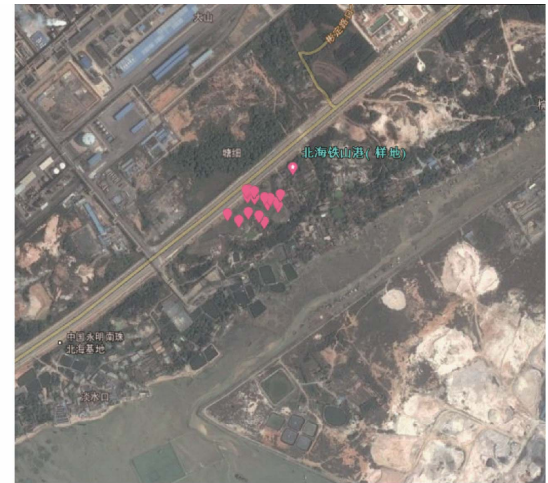

港口 Port

图4 农田、湿地、港口3种典型生态系统外来入侵植物普查样地和数据采集点空间分布

Fig. 4 Spatial distribution of sample plot and data collection points of invasive alien plants survey in three typical ecosystems of farmland, wetland and port

分布与资源库建设”和福建省科技重大专项“重大外 来入侵生物绿色防控综合技术研究与示范”的调查 任务, 2017年3-5月分别在福建、广西、云南三省应 用调查规划方法开展调查。针对农田、湿地、港口 3种生态类型, 以三省主要高速公路为代表性调查 线路, 设置 $35 \mathrm{~km} \times 40 \mathrm{~km}$ 调查地理网格, 结合各省 具体情况分别规划外来入侵植物普查样地。共计规 划普查样地 61 个, 样地间平均间隔 $30 \mathrm{~km}$, 普查区域 覆盖41个县域 43 个调查地理网格。

三省外来入侵植物普查总计采集调查数据 804 条, 涉及植物种类累计 96 种, 调查直线距离达 $1,856 \mathrm{~km}$, 三省普查工作统计如表3所示。通过前期 的调查规划, 平均每日可调查 6 个样地, 采集调查 数据 80 多条, 调查直线距离达 $180 \mathrm{~km}$, 每个样地的 导航精度高于 $15 \mathrm{~m}$ 。通过Excel软件对三省普查数据 进行汇总整理，运用LocaSpace Viewer软件的“Excel 转KML”功能生成调查数据空间分布的KML文件, 在LocaSpace Viewer软件中进行浏览。图4显示了在 农田、湿地、港口 3 种典型生态系统中外来入侵植
物普查样地和数据采集点的空间分布情况。

\section{2 对比实验}

2017年3月26-27日，在广西外来入侵植物普查 中基于规划调查与无规划调查开展对比实验。分成 2 个调查小组, 一组在调查前对调查区域、调查路 线、调查样地进行规划, 一组则无规划开展调查。 每个调查小组 4 名成员, 驾车前往各调查样地, 每 天调查 8 小时。对两组调查数据进行处理分析, 通过 调查覆盖县域、覆盖地理网格数量、调查样地数量 和调查覆盖生境类型衡量调查范围, 通过调查样地 间平均直线距离和标准差衡量样地空间分布的均 一性，通过调查样地间行程平均花费时间和标准差 衡量调查时间利用效率。无规划的样地与基于规划 的样地空间分布如图5所示。无规划的调查与基于 规划的调查指标数据比较如表4所示。

\section{5 结论}

2017年3-5月在福建、广西、云南外来入侵植 物普查中开展的技术方法应用与对比实验结果表 

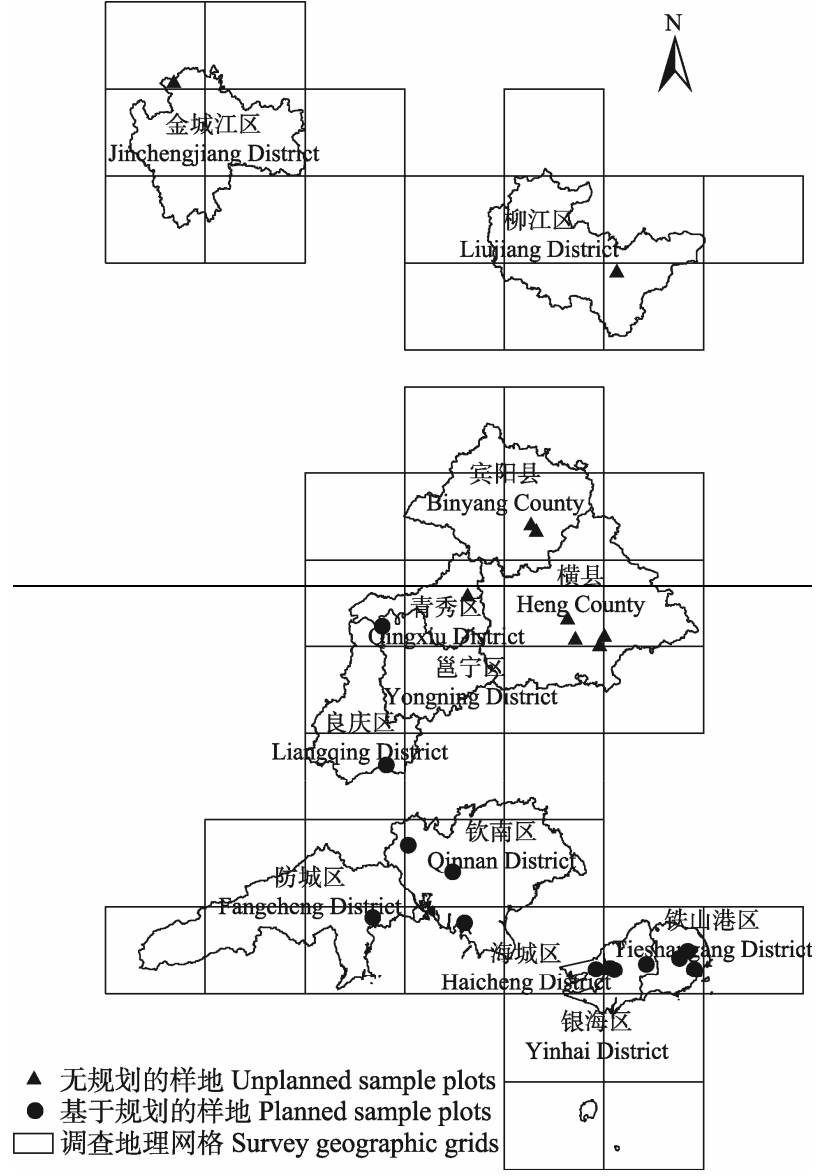

图5 无规划的样地与基于规划的样地空间分布

Fig. 5 The spatial distribution of unplanned sample plots and planned sample plots

明, 在同等条件下, 运用GIS技术进行前期调查规 划能够使调查工作更加规范、有序; 调查实施更具 针对性; 调查覆盖范围更广; 调查生境类型更丰富; 调查样地数量更多, 在空间分布上更均匀和科学, 避免了随机设置样地影响调查数据的分析与应用; 极大降低了野外寻找、挑选调查样地花费的人力和 时间成本, 调查时间利用效率提高 1 倍。可为跨区 域、长时间、多单位协作开展外来入侵植物调查提 供技术支撑, 为外来入侵物种分布与扩散调查提供 科学、高效的技术解决方案。

外来入侵植物调查作为外来入侵物种调查的 组成部分, 其规程和技术方法与入侵动物和入侵病 原微生物调查有着相似之处, 其他入侵物种调查可 参照本文提出的技术方法, 根据调查区域的实际情 况调整调查点及周边踏查范围、样方大小、调查点 或样地空间间隔等指标即可满足调查规划的要求。
表4 无规划调查与基于规划的调查指标数据比较

Table 4 Comparison of target data for unplanned survey and planned survey

\begin{tabular}{|c|c|c|}
\hline & $\begin{array}{l}\text { 无规划调查 } \\
\text { Unplanned } \\
\text { survey }\end{array}$ & $\begin{array}{l}\text { 基于规划的调查 } \\
\text { Planned survey }\end{array}$ \\
\hline $\begin{array}{l}\text { 覆盖县域数 } \\
\text { Number of covered counties }\end{array}$ & 5 & 7 \\
\hline $\begin{array}{l}\text { 覆盖调查地理网格数 } \\
\text { Number of covered geographic grids }\end{array}$ & 6 & 7 \\
\hline $\begin{array}{l}\text { 调查样地数量 } \\
\text { Number of sample plots }\end{array}$ & 9 & 13 \\
\hline $\begin{array}{l}\text { 调查区生境类型 } \\
\text { Habitat types in the survey area }\end{array}$ & $\begin{array}{l}\text { 河岸、农田 } \\
\text { Riverside, } \\
\text { farmland }\end{array}$ & $\begin{array}{l}\text { 农田、港口、高 } \\
\text { 速公路沿线、荒 } \\
\text { 地、公园、园林 } \\
\text { 绿化 Farmland, } \\
\text { Ports, areas along } \\
\text { the expressway, } \\
\text { wasteland, parks, } \\
\text { landscaping }\end{array}$ \\
\hline $\begin{array}{l}\text { 调查点间平均直线距离 } \\
\text { Average direct distance between } \\
\text { survey points }(\mathrm{km})\end{array}$ & 53.5 & 30.5 \\
\hline $\begin{array}{l}\text { 调查点间直线距离标准差 } \\
\text { Standard deviation of direct distance } \\
\text { between survey points }(\mathrm{km})\end{array}$ & 68.2 & 27.7 \\
\hline $\begin{array}{l}\text { 调查点间行程平均花费时间 } \\
\text { Average cost of travel time between } \\
\text { survey points (min) }\end{array}$ & 128 & 66 \\
\hline $\begin{array}{l}\text { 调查点间行程花费时间标准差 } \\
\text { Standard deviation of travel time } \\
\text { between survey points (min) }\end{array}$ & 127 & 36 \\
\hline
\end{tabular}

同时，本文提出的技术方法同样适用于其他生物多 样性野外调查工作, 但是在调查规划中需要根据不 同的调查规程、对象和区域，对分析方法和指标作 出相应的调整，以满足不同调查的需要。

由于时间、人员和技术等因素限制，本文仅对 入侵植物普查进行验证与应用。在调查规划中还可 以结合气候、物种适生性分布、人文经济等多种背 景数据, 让调查规划更有针对性, 能够应对更为复 杂的研究需求。以上不足将在后续的研究工作中进 行补充与完善。

致谢：感谢中国农业科学院植物保护研究所、广西 农业科学院植物保护研究所、云南农业大学对本研 究项目给予的大力支持并参与相关工作。感谢张毅 波、陈红松、王彦辉、桂富荣、喜超、李建宇、史 梦竹等参与实地调查工作。感谢周忠实研究员在方 法构建中提出的宝贵意见。 


\section{参考文献}

Chen B (2016) Geographic data management, refined route design and precise navigation in biodiversity field surveys. Biodiversity Science, 24, 701-708. (in Chinese with English abstract) [陈涁 (2016) 生物多样性野外调查地理信息管 理、路线精细设计和精确导航方法. 生物多样性, 24 , 701-708.]

Ding H, Ma FZ, Wu J, Lei JC, Le ZF, Xu HG (2015) Considerations of building up a supervision and management system for prevention and control of invasive alien species posing environmental hazards in China. Journal of Ecology and Rural Environment, 31, 652-657. (in Chinese with English abstract) [丁晖，马方舟，吴军，雷军成，乐志芳，徐 海根 (2015) 关于构建我国外来入侵物种环境危害防控 监督管理体系的思考. 生态与农村环境学报，31, 652-657.]

Fang JY, Wang XP, Shen ZH, Tang ZY, He JS, Yu D, Jiang Y, Wang ZH, Zheng CY, Zhu JL, Guo ZD (2009) Methods and protocols for plant community inventory. Biodiversity Science, 17, 533-548. (in Chinese with English abstract) [方精 云, 王襄平, 沈泽吴, 唐志尧, 贺金生, 于丹, 江源, 王志 恒, 郑成洋, 朱江玲, 郭兆迪 (2009) 植物群落清查的主 要内容、方法和技术规范. 生物多样性, 17, 533-548.]

Ma LG, Cao YR (2010) Application of Google Earth COM API and KML in tourism information system development. Journal of Geo-Information Science, 12, 828-834. (in Chinese with English abstract) [马立广, 曹彦荣 (2010) Google Earth COM API及KML技术在旅游管理信息系统
开发中的应用. 地球信息科学学报, 12, 828-834.]

McMaugh T (translated by Department of Biological Invasions, Institute of Plant Protection, Chinese Academy of Agricultural Sciences) (2013) Guidelines for Surveillance for Plant Pests in Asia and the Pacific. Science Press, Beijing. (in Chinese) [中国农业科学院植物保护研究所生物入侵研究 室(译) (2013) 亚太地区植物有害生物监控指南. 科学出 版社, 北京.]

Sun WT, Liu YT (2010) Research progress of risk analysis of biological invasion. Chinese Agricultural Science Bulletin, 26, 233-236. (in Chinese with English abstract) [孙文涛, 刘雅婷 (2010) 生物入侵风险分析的研究进展. 中国农 学通报, 26, 233-236.]

Wan FH, Feng J, Xu J (2011) Biological Invasions: Detection, Surveillance and Monitoring. Science Press, Beijing. (in Chinese) [万方浩, 冯洁, 徐进 (2011) 生物入侵: 检测与 监测篇. 科学出版社, 北京.]

Wan FH, Guo JY, Wang DH (2002) Alien invasive species in China: Their damages and management strategies. Biodiversity Science, 10, 119-125. (in Chinese with English abstract) [万方浩, 郭建英, 王德辉 (2002) 中国外来入侵生 物的危害与管理对策. 生物多样性, 10, 119-125.]

Yang LF (2013) Geographic data capturing technique based on Google Maps API V3. Remote Sensing Technology and Application, 28, 791-798. (in Chinese with English abstract) [杨立法 (2013) 基于Google Maps API V3的地理数据采 集技术. 遥感技术与应用, 28, 791-798.]

(责任编辑：黄祥忠)

\section{附录 Supplementary Material}

附录1 基于GIS技术的外来入侵植物普查规划与外业实施技术路线

Appendix 1 The technical route of overall survey planning and field operation of alien invasive plants based on GIS technology http://www.biodiversity-science.net/fileup/PDF/2017314-1.pdf

附录2 用于调查地理网格贴图的十字透明背景纹理图片

Appendix 2 Cross-shaped texture picture with transparent background used to structure survey geographic grids http://www.biodiversity-science.net/fileup/PDF/2017314-2.pdf 
陈宏，冼晓青，邱荣洲，池美香，赵健. 基于GIS的外来入侵植物调查规划与外业实施. 生物多样性, 2018, 26 (1): 44-52.

http://www.biodiversity-science.net/CN/10.17520/biods.2017314

\section{附录1 基于GIS技术的外来入侵植物普查规划与外业实施技术路线}

Appendix 1 The technical route of overall survey planning and field operation of alien invasive plants based on GIS technology

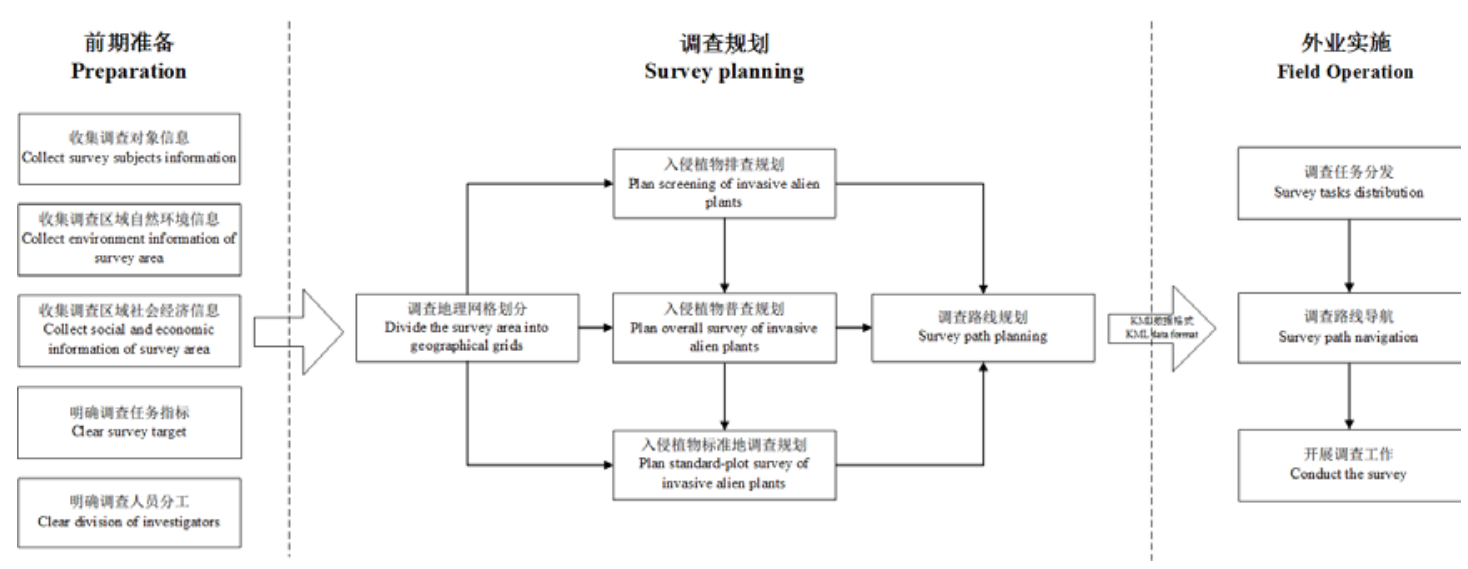


陈宏，冼晓青，邱荣洲，池美香，赵健. 基于GIS的外来入侵植物调查规划与外业实施. 生物多样性, 2018, 26 (1): 44-52.

http://www.biodiversity-science.net/CN/10.17520/biods.2017314

附录2 用于调查地理网格贴图的十字透明背景纹理图片

Appendix 2 Cross-shaped texture picture with transparent background used to structure survey geographic grids

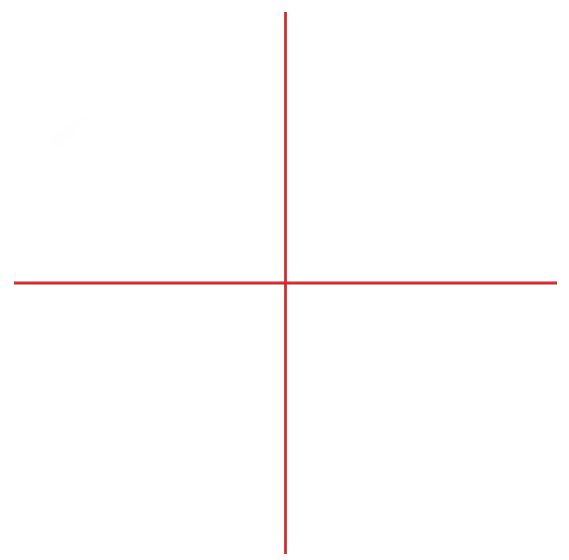

